Gynäkologische Endokrinologie 2014 .

12:119-123

DOI 10.1007/s10304-014-0631-5

Online publiziert: 6. April 2014

(c) Springer-Verlag Berlin Heidelberg 2014

\section{Redaktion}

L. Kiesel, Münster

A.O. Mueck, Tübingen

P. Stute, Bern

L. Kramer · J. Füri · P. Stute

Abteilung für Gynäkologische Endokrinologie und Reproduktionsmedizin,

Frauenklinik, Inselspital Bern, Schweiz

\title{
Die gesundheitsbezogene Lebensqualität
}

what to do about" [4]. Im Jahr 1994 erschien gar eine Publikation mit dem plakativen Titel „Let's hang up 'quality of life as a hopeless term" [5].

\section{Ist eine Definition von Lebensqualität möglich?}

In der wissenschaftlichen Literatur werden viele Begriffe als Synonym zur Lebensqualität gebraucht. Wohlstand, (Lebens-)Zufriedenheit, subjektives Wohlbefinden, funktionaler Status, der Gesundheitszustand, das Glück sowie „the good life" werden zunehmend gleichwertig verwendet. In der deutschsprachigen Literatur wird Lebensqualität insbesondere mit dem Begriff Wohlbefinden gleichgesetzt [6]. Wie bereits semantisch offensichtlich, ist eine klare Abgrenzung beider Konzepte kaum möglich. Diese unscharfe Trennlinie stellt einen ersten Schwierigkeitsfaktor für eine eindeutige Definition des Begriffs Lebensqualität dar.

Zusätzlich besteht bislang keine Einigkeit, ob die Lebensqualität nur subjektiv zu bestimmen ist oder ob auch objektive Komponenten eine Rolle spielen. So schreiben die Autoren in der Einleitung zum Inventar zur Erfassung der Lebensqualität bei Kindern und Jugendlichen: „Lebensqualität im engeren Sinne umfasst zwei grundlegend unterschiedliche Grundaspekte: Einerseits die objektive Handlungs- und Funktionsfähigkeit und andererseits das subjektive Wohlbefinden bzw. die subjektive Zufriedenheit mit der eigenen körperlichen und psychischen Verfassung, Lebenssituation und Lebensführung“ [7]. Mehrheitlich wird der Begriff Lebensqualität allerdings auf die subjektive Lebensqualität beschränkt.
Die World Health Organization Quality of Life (WHOQOL) Group geht davon aus, dass ein entscheidendes Charakteristikum des Konstrukts Lebensqualität die Subjektivität sei [8].

> Eine eindeutige Definition der Lebensqualität gibt es bis heute nicht.

Deshalb werden mit den zahlreich entwickelten Messinstrumenten auch ganz unterschiedliche Domänen erfasst [9]:

- der allgemeine Gesundheitszustand,

- die funktionellen Fähigkeiten,

- die emotionale Funktion,

- das Niveau des Wohlbefindens,

- die Zufriedenheit mit dem Leben,

- die intellektuelle Ebene,

- Schmerzen,

- Übelkeit, Erbrechen und andere Symptome,

- die sexuelle Funktion,

- die soziale Aktivität und

- der berufliche Status.

Da das Verständnis der Lebensqualität von Mensch zu Mensch variiert, haben die zahlreichen Domänen, die mit den Instrumenten gemessen oder erfragt werden, für jede Person eine andere Bedeutung und Gewichtung. M. Radoschewski vom Robert Koch-Institut in Berlin hält fest, dass „unabhängig davon, welchem definitorischen Ansatz von Lebensqualität man zuneigt, 'Lebensqualität' oder auch nur 'gesundheitsbezogene Lebensqualität' als theoretische Konzepte so komplex und multidimensional sind, dass es a priori nicht möglich sein kann, sie empirisch erschöpfend, sondern allenfalls hinreichend (für ein bestimmtes Untersuchungsziel) $\mathrm{zu}$ erfassen, $\mathrm{zu}$ beschreiben und $\mathrm{zu}$ ana- 


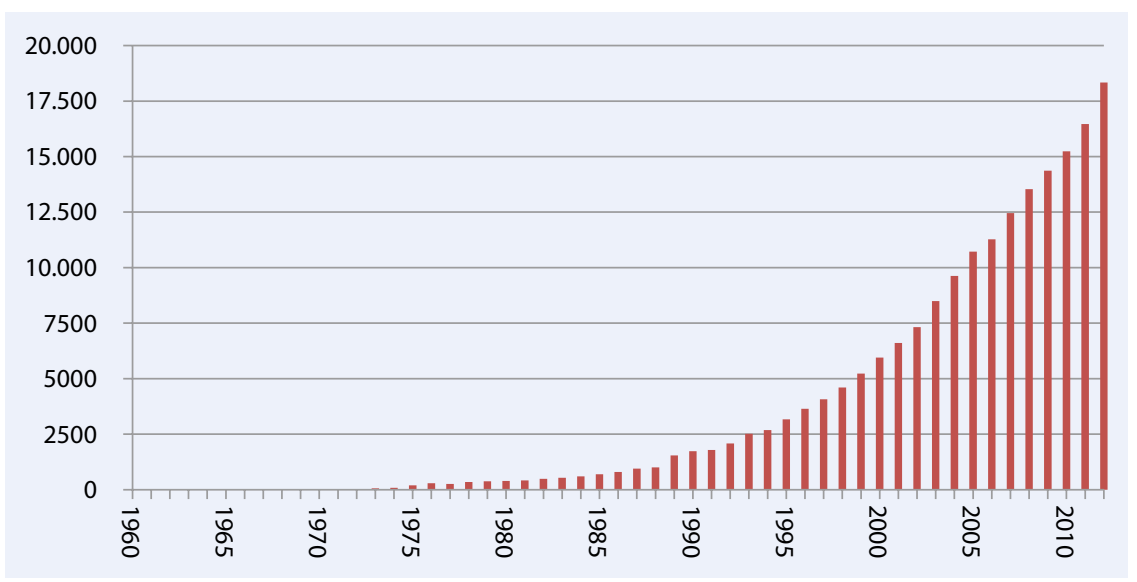

Abb. 1 A PubMed-Publikationen ab 1960 zum Stichwort "quality of life"

lysieren. Damit sind auch Grenzen für die Entwicklung und Nutzung darauf ausgerichteter Messinstrumente gesetzt" [10].

Die WHOQOL Group legte 1993 wohl eine der bislang komplexesten Definitionen vor. Die Arbeitsgruppe definiert Lebensqualität „als die subjektive Wahrnehmung einer Person über ihre Stellung im Leben in Relation zur Kultur und den Wertsystemen, in denen sie lebt, und in Bezug auf ihre Ziele, Erwartungen, Standards und Anliegen. Es handelt sich um ein Arbeitskonzept, das in komplexer Weise beeinflusst wird durch die körperliche Gesundheit, den psychologischen $\mathrm{Zu}$ stand, den Grad der Unabhängigkeit, die sozialen Beziehungen und den hervorstechenden Eigenschaften der Umwelt. Hierbei werden mehrere Domänen (körperlich, psychologisch, sozial, funktional, umweltbezogen und werthaltungsbezogen) und darin lokalisierte Facetten einbezogen“" [11].

\section{Instrumente zur Erfassung der Lebensqualität}

Die Anzahl der Publikationen zum Begriff der Lebensqualität ist in den letzten 20 Jahren explosionsartig gestiegen (- Abb. 1). Bevor die Bedeutung des neuen Outcomes definiert wurde, entwickelten Wissenschaftler unzählige Messinstrumente, die teilweise bereits vielfach in der Klinik oder Forschung eingesetzt werden. Radoschewski stellt fest: „In der einschlägigen gesundheitsorientierten Fachliteratur zur Thematik Lebensqualität bzw. Messung von Lebensqualität dominieren Publikationen zu instrumentel- len Entwicklungen, zu deren Erprobung und methodischen Überprüfung, gegenüber theoretisch-konzeptionell orientierten“ [10].

Während einige Autoren argumentieren, dass „objektive Gesundheitsindikatoren, wie physiologische Kennwerte oder auch Fremdurteile von Ärzten, nur geringe bis gar keine Korrelationen mit der subjektiven Lebensqualität zeigen“" [12], sind andere der Auffassung, dass die Lebensqualität in zwei Gebiete unterteilt werden sollte: in die objektive Handlungs- und Funktionsfähigkeit und in das subjektive Wohlbefinden bzw. die subjektive Zufriedenheit [7]. Auch Pöthig et al. [13] betrachten die Lebensqualität als ein Konstrukt, das objektiv messbare Wirkungen des Alltagsverhaltens beinhalten sollte: „Parameter der Vitalität und gesundheitsbezogenen Lebensqualität werden bisher meist im Rahmen rein subjektiver Einschätzungen durch den Patienten anhand von Fragebogen oder strukturierter Interviews ermittelt. Diese Vorgehensweise ersetzt keinesfalls eine valide Messung von Parametern und Indikatoren, die eine differenzierte Aussage über die objektiv messbaren Wirkungen des Alltagsverhaltens auf das Biosystem erlaubt. Auch die Wirksamkeit von medizinischen oder gesundheitsförderlichen Interventionen auf die Funktionalität des soziobiologischen Gesamtsystems des Menschen lässt sich mit Fragebögen allein nicht hinreichend erfassen." Wenn auch im aktuellen Diskurs mehrheitlich die Subjektivität des Konstrukts Lebensqualität betont wird, scheinen die Verfechter eines zwei- oder mehrteiligen
Verständnisses menschlicher Lebensqualität, die sowohl subjektiven als auch objektiven Parametern Raum geben, an Boden gut zu machen.

\section{Die gesundheitsbezogene Lebensqualität (HRQoL)}

In der Medizin und in klinischen Studien wird der sehr breite und übergreifende Ansatz von Lebensqualität meist etwas eingeengt. Die in der Medizin relevante Lebensqualität unterscheidet sich von der allgemeinen Lebensqualität, die durch Lebensbedingungen wie die Verfügbarkeit von Nahrung, Wohnraum, Verbrauchsgütern und Genussmitteln, durch den Lebensstandard und letztlich auch durch unseren Gesundheitszustand bestimmt wird. Aus medizinischer Sicht interessiert aus diesem weiten Bereich der allgemeinen Lebensqualität lediglich ein Sektor, nämlich die gesundheitsbezogene Lebensqualität ["health-related quality of life" (HRQoL)]. Auch hier liegt keine eindeutige Definition vor, weshalb man sich an der Definition des Begriffs Gesundheit der WHO von 1946 orientiert: „Health is a state of complete physical, mental, and social well-being and not merely the absence of disease or infirmity" [14].

\section{》) Die gesundheitsbezogene Lebensqualität ist ein mehrdimensionales Konzept}

Die gesundheitsbezogene Lebensqualität bezieht sich auf diese Gesundheitsdefinition und ist somit auch ein mehrdimensionales Konzept. Es wird nicht nur die physische Komponente der Gesundheit einbezogen, d. h. Symptome, sondern auch die soziale und psychische Dimension. „Das Gesundheitskonzept ist", so B. Lindström, „noch immer zu stark mit Krankheit verknüpft und in der Forschung zu wenig mit Konzepten verbunden, die von positiven Werten ausgehen, wie etwa das Salutogenese-Konzept. Lebensqualität ist ein solcher positiver Ansatz" [10].

Schließlich besteht auch auf dem Gebiet der gesundheitsbezogenen Lebensqualitätsforschung ein weitreichender Konsens in der Bevorzugung von Infor- 
mationen, die von der betroffenen (kranken) Person selbst stammen und nicht vom behandelnden Arzt, im Sinne einer Vermutung oder Fremdeinschätzung [15].

\section{Die Bedeutung der HRQoL-Messung in der Medizin}

Die ehemals im Fokus stehende Heilung von Krankheiten und die Verlängerung der Lebensdauer sind nun der Linderung von Beschwerden gewichen. Damit ist die Erfassung und Erhaltung der gesundheitsbezogenen Lebensqualität in der klinischen Forschung zur dritten Säule neben Wirksamkeit (Effektivität) und Nebenwirkungen geworden [16]. In der Bevölkerung, in der Literatur und auch aus ärztlicher Sicht herrscht Konsens darüber, dass die Erhaltung oder Verbesserung der Lebensqualität für sich ein wesentliches Behandlungs- bzw. Lebensziel darstellt, das keiner weiteren Legitimation bedarf [3]. Auch wenn die Überlebenslänge sich nicht verändert, kann sich dennoch die Lebensqualität verbessern oder verschlechtern [17].

\ Die Messung der Lebensqualität oder HRQoL hat in den letzten Jahren stark an Popularität gewonnen.

Sie gibt den Personen, die Pflege erhalten, eine Stimme und reflektiert die Gedanken und Gefühle der Menschen, die eine Krankheit oder eine Behandlung durchleben. Sie ist aber auch ein Indikator dafür, ob das System der Interventionen funktioniert. HRQoL-Messungen erlauben es den Patienten, ihre individuellen Werte und Präferenzen in die Beurteilung einfließen zu lassen [18].

Folgende Aspekte sind in der klinischen Praxis relevant [19]: Zum einen profitiert der Patient von den Ergebnissen klinischer Studien, insbesondere von psychosozialen Interventionen, die auf der Basis von Diskussionen zur Lebensqualität oder von Forschungsergebnissen entwickelt worden sind, zum anderen profitiert er auch von Ärzten mit verändertem Bewusstsein, die für das psychosomatische Gesamtbefinden der Patienten sensibilisiert sind und die Arzt-Patienten-Beziehung neu gestalten.

\section{Herausforderungen bei der Erfassung der (gesundheits- bezogenen) Lebensqualität}

Die Messung der Lebensqualität - und auch die der HRQoL - wird durch den Umstand erschwert, dass Lebensqualität von jeder Person anders interpretiert wird. Verschiedene Komponenten spielen je nach Person eine mehr oder weniger wichtige Rolle. Die einzelnen Fragen werden unterschiedlich verstanden und gewichtet. Insbesondere wenn verschiedene Kulturen und verschiedene soziale Hintergründe aufeinandertreffen, hat Lebensqualität heterogene Bedeutungen. Subjektive Lebensqualitätswerte können zudem auch stark durch Persönlichkeitsfaktoren beeinflusst werden [20]. Und obwohl einige Autoren eine objektive Messung grundsätzlich begrüßen würden, gibt es kaum solche Messverfahren.

Trotz der Schwierigkeiten im Umgang mit der Lebensqualität, lässt sich das Konstrukt aus der Wissenschaft nicht mehr wegdenken. „Die mittlerweile selbstverständlich gewordene Erhebung von Lebensqualitätsdaten hat dazu geführt, dass die Anwendung bereits bekannter Verfahren explosionsartig gestiegen ist, während grundsätzliche Fragen der Konzeptualisierung von gesundheitsbezogener Lebensqualität und der Validität der verwandten Messinstrumente ins Hintertreffen geraten sind" [21]. Die Multidimensionalität des Begriffs führte da$\mathrm{zu}$, dass allerlei Instrumente entwickelt wurden. Das große Spektrum verschiedener Instrumente stellt ein großes Problem in der Erforschung der Lebensqualität dar. Es ist kaum möglich, zwei Instrumente miteinander zu vergleichen; eine Interpretation wird schwierig oder gar unmöglich [22].

In - Tab. 1 sind die wichtigsten und am weitesten verbreiteten Instrumente zur Erfassung der Lebensqualität aufgeführt. Bei der Erstellung wurde auf die umfassende Sammlung psychodiagnostischer Verfahren zur Erfassung der gesundheitsbezogenen Lebensqualität von J. Schumacher, A. Klaiberg u. E. Brähler zurückgegriffen [6]. Aus den über 70 Verfahren wurde anhand bestimmter Leitkriterien eine Auswahl getroffen. So fanden nur generische Instrumente Berücksichti-
Gynäkologische

Endokrinologie 2014 · 12:119-123

DOI 10.1007/s10304-014-0631-5

c) Springer-Verlag Berlin Heidelberg 2014

\section{Kramer · J. Füri · P. Stute Die gesundheitsbezogene Lebensqualität}

\section{Zusammenfassung}

Die Messung der Lebensqualität gewinnt zunehmend an Bedeutung. Obwohl eine Definition des Begriffs fehlt, wurden unzählige Messinstrumente zu ihrer Erfassung entwickelt, vorwiegend subjektive Fragebogen. In der Medizin wird die Lebensqualität eingeschränkt, man spricht von der gesundheitsbezogenen Lebensqualität ["health-related quality of life" (HRQoL)]. Ihre Messung ist heute Teil der Beurteilung von medizinischen Interventionen. Im vorliegenden Beitrag wird das Konstrukt der (gesundheitsbezogenen) Lebensqualität beleuchtet und eine Auswahl von Instrumenten zur Erfassung der HRQoL vorgestellt. Herausforderungen im Umgang mit der HRQoL werden besprochen und der Einbezug von objektiv gemessenen Parametern diskutiert.

Schlüsselwörter

Lebensqualität · Definition · Diagnostische Verfahren · Subjektive Messung · Objektive Messung

\section{Health-related quality of life}

\section{Abstract}

The assessment of quality of life ( $Q \mathrm{QL}$ ) has become very popular during recent years. Despite the lack of a definition of the term, countless instruments for measuring QoL have been developed, mainly as subjective questionnaires. In medicine, the QoL is mostly limited to health-related QoL (HRQoL) and its assessment has increasingly become part of clinical trials. In this article the construct of (HR) QoL is illuminated and a selection of instruments for HRQoL assessment is presented. Challenges in dealing with HRQoL are discussed and the inclusion of objectively measured parameters is discussed.

\section{Keywords}

Quality of life - Definition - Diagnostic

techniques and procedures - Subjective assessment · Objective assessment

gung. Die Evidenz für Reliabilität und Validität musste empirisch belegt sein. Eine weitere Voraussetzung war, dass das Verfahren zugleich in einer deutschen und englischen Fassung vorliegt und bei Er- 
Tab. 1 Instrumente zur Erfassung der Lebensqualität

\begin{tabular}{|c|c|c|c|}
\hline Name & Art der Erfassung & Erfasste Kategorien & Einsatzgebiete \\
\hline $\begin{array}{l}\text { Affect Balance Scale } \\
\text { (ABS) }\end{array}$ & $\begin{array}{l}\text { 2-dimensionales Selbst- } \\
\text { beurteilungsverfahren; } \\
\text { 4-stufiges Antwortmodell }\end{array}$ & $\begin{array}{l}10 \text { Items zur Erfassung des emotionalen Wohlbefindens } \\
\text { [9]; } \\
2 \text { Skalen: positives emotionales Befinden, negatives } \\
\text { emotionales Befinden; } \\
\text { nur Häufigkeit, keine Intensität }\end{array}$ & $\begin{array}{l}\text { Erwachsene; } \\
\text { klinisch/nichtklinisch; } \\
\text { gerontologische Forschung }\end{array}$ \\
\hline $\begin{array}{l}\text { Befindlichkeitsskalen } \\
\text { zur Messung von ak- } \\
\text { tueller Stimmung und } \\
\text { Grundgestimmtheit } \\
\text { (BFS) }\end{array}$ & 5-stufiges Antwortmodell & $\begin{array}{l}40 \text { Items (Adjektivliste); } \\
8 \text { Skalen: Aktiviertheit, Erregtheit, gute Laune, Ruhe, } \\
\text { Energielosigkeit, Ärger, Deprimiertheit, Besinnlichkeit }\end{array}$ & $\begin{array}{l}\text { Ab } 14 \text { Jahren; } \\
\text { Stimmung im Kontext körperlicher } \\
\text { und sportlicher Aktivitäten }\end{array}$ \\
\hline $\begin{array}{l}\text { Befindlichkeits-Skala } \\
\text { (Bf-S, Bf-S')/Adjective } \\
\text { Mood Scale (AMS, } \\
\text { AMS') }\end{array}$ & $\begin{array}{l}\text { Fragebogen zur Selbst- } \\
\text { beurteilung }\end{array}$ & $\begin{array}{l}28 \text { Gegensatzpaare, Bestimmung der momentanen } \\
\text { Befindlichkeit }\end{array}$ & Erwachsene \\
\hline $\begin{array}{l}\text { European Quality of } \\
\text { Life Questionnaire } \\
\text { (EuroQoL, EQ-5D) }\end{array}$ & $\begin{array}{l}\text { Mehrdimensionales Selbst- } \\
\text { beurteilungsverfahren; } \\
\text { 3-stufiges Antwortmodell }\end{array}$ & $\begin{array}{l}5 \text { Items; } \\
5 \text { Dimensionen: Beweglichkeit/Mobilität, für sich selbst } \\
\text { sorgen, allgemeine Tätigkeiten, Schmerzen/körperliche } \\
\text { Beschwerden, Angst/Niedergeschlagenheit }\end{array}$ & $\begin{array}{l}\text { Erwachsene; } \\
\text { gesundheitsökonomische Studien, } \\
\text { klinische Untersuchungen, phar- } \\
\text { makologische Untersuchungen, } \\
\text { Gesundheitsumfragen }\end{array}$ \\
\hline $\begin{array}{l}\text { Eigenschaftswörter- } \\
\text { liste (EWL) }\end{array}$ & $\begin{array}{l}\text { Mehrdimensionales Selbst- } \\
\text { beurteilungsverfahren }\end{array}$ & $\begin{array}{l}\text { Beschreibung des momentanen Befindens anhand von } \\
\text { Eigenschaftswörtern, je nach Fragebogenform zwi- } \\
\text { schen } 40 \text { und } 160 \text { Eigenschaftswörter; } \\
5 \text { Kombinationsskalen: leistungsbezogene Aktiviertheit, } \\
\text { Wohlbehagen, allgemeine Desaktiviertheit, allgemeine } \\
\text { Gereiztheit, Angst/Deprimiertheit }\end{array}$ & $\begin{array}{l}\text { Gesunde/Kranke, 14- bis } 80 \text {-jährig; } \\
\text { wiederholte Erfassung von Be- } \\
\text { findenszuständen und Zustands- } \\
\text { schwankungen }\end{array}$ \\
\hline $\begin{array}{l}\text { Fragebogen zur Er- } \\
\text { fassung des aktuellen } \\
\text { körperlichen Wohl- } \\
\text { befindens (FAW) }\end{array}$ & $\begin{array}{l}\text { Mehrdimensionales Selbst- } \\
\text { beurteilungsverfahren }\end{array}$ & $\begin{array}{l}58 \text { Items; } \\
7 \text { Skalen: Zufriedenheit mit dem gegenwärtigen } \\
\text { Körperzustand, Ruhe und Muse, Vitalität und Lebens- } \\
\text { freude, nachlassende Anspannung, Genussfreude } \\
\text { und Lustempfinden, subjektive Konzentrations- und } \\
\text { Reaktionsfähigkeit, Gepflegtheit/Frische/angenehmes } \\
\text { Hautempfinden }\end{array}$ & $\begin{array}{l}\text { Erwachsene; } \\
\text { Gesundheitspsychologie, Verhal- } \\
\text { tensmedizin, Psychotherapie }\end{array}$ \\
\hline $\begin{array}{l}\text { Habituelle subjektive } \\
\text { Wohlbefindensskala } \\
\text { (HSWBS) }\end{array}$ & $\begin{array}{l}\text { 2-dimensionales Selbst- } \\
\text { beurteilungsverfahren; } \\
\text { 6-stufiges Antwortverfahren }\end{array}$ & $\begin{array}{l}13 \text { Items; } \\
2 \text { Skalen: Stimmungsniveau, allgemeine Lebenszufrie- } \\
\text { denheit }\end{array}$ & Jugendliche und Erwachsene \\
\hline $\begin{array}{l}\text { Kieler Interview zur } \\
\text { subjektiven Situa- } \\
\text { tion - Belastungen/ } \\
\text { Ressourcen (KIsS-BR) }\end{array}$ & $\begin{array}{l}\text { Selbstbeurteilungsverfahren; } \\
\text { Interview- oder Fragebogen- } \\
\text { version; } \\
\text { 7-stufiges Antwortverfahren }\end{array}$ & $\begin{array}{l}15 \text { verschiedene Lebensbereiche aus den Bereichen: } \\
\text { Arbeit, Partnerschaft, Familie, soziale Beziehungen, } \\
\text { Freizeit, Finanzen, in Bezug zu erlebten Belastungen } \\
\text { und Ressourcen }\end{array}$ & $\begin{array}{l}\text { Krankheitsübergreifend bzw. } \\
\text { unabhängig von einer Krankheits- } \\
\text { situation }\end{array}$ \\
\hline $\begin{array}{l}\text { Der Mehrdimensio- } \\
\text { nale Befindlichkeits- } \\
\text { Fragebogen (MDBF) }\end{array}$ & $\begin{array}{l}\text { Selbstbeurteilungsverfahren; } \\
\text { 5-stufiges Antwortverfahren }\end{array}$ & $\begin{array}{l}24 \text { Items; } \\
3 \text { Skalen: gute/schlechte Stimmung, Wachheit/Müdig- } \\
\text { keit, Ruhe/Unruhe }\end{array}$ & $\begin{array}{l}\text { Jugendliche und Erwachsene; } \\
\text { Therapieevaluation, Grundlagen- } \\
\text { forschung }\end{array}$ \\
\hline $\begin{array}{l}\text { Nottingham Health } \\
\text { Profile (NHP) }\end{array}$ & $\begin{array}{l}\text { Mehrdimensionales Selbst- } \\
\text { beurteilungsverfahren; } \\
\text { dichotomes Antwort- } \\
\text { verfahren }\end{array}$ & $\begin{array}{l}38 \text { Items; } \\
6 \text { Skalen: Energieverlust, Schmerz, emotionale Reaktion, } \\
\text { Schlaf, soziale Isolation, physische Mobilität }\end{array}$ & $\begin{array}{l}\text { Screening zur Identifikation von } \\
\text { Personen mit mittel- bis schwergra- } \\
\text { digen Beeinträchtigungen, Evalua- } \\
\text { tionsstudien }\end{array}$ \\
\hline $\begin{array}{l}\text { Positive and Nega- } \\
\text { tive Affect Schedule } \\
\text { (PANAS) }\end{array}$ & $\begin{array}{l}\text { Fragebogen zur Selbst- und } \\
\text { Fremdbeurteilung; } \\
\text { 5-stufiges Antwortverfahren }\end{array}$ & $\begin{array}{l}20 \text { Items; } \\
2 \text { Skalen: positiver Affekt, negativer Affekt }\end{array}$ & $\begin{array}{l}\text { Bereichsübergreifendes Erhebungs- } \\
\text { verfahren für den Erwachsenen- } \\
\text { bereich }\end{array}$ \\
\hline $\begin{array}{l}\text { Psychological General } \\
\text { Well-Being Index } \\
\text { (PGWBI) }\end{array}$ & $\begin{array}{l}\text { Fragebogen zur subjektiven } \\
\text { Einschätzung des Wohl- } \\
\text { befindens; } \\
\text { 6-stufiges Antwortverfahren }\end{array}$ & $\begin{array}{l}22 \text { Items; } \\
6 \text { Skalen: Angst, Depression, Wohlbefinden, Selbst- } \\
\text { kontrolle, Gesundheit, Vitalität }\end{array}$ & $\begin{array}{l}\text { Erwachsene, Gesunde/Kranke; } \\
\text { klinisch/nichtklinisch }\end{array}$ \\
\hline $\begin{array}{l}\text { Profile of Mood States } \\
\text { (POMS) }\end{array}$ & $\begin{array}{l}\text { Fragebogen zur Selbst- } \\
\text { einschätzung; } \\
\text { 5-stufiges Antwortverfahren }\end{array}$ & $\begin{array}{l}65 \text { Items; } \\
7 \text { Skalen: Niedergeschlagenheit, Angst, Müdigkeit, } \\
\text { Tatkraft, Missmut, Spannung, Verwirrung }\end{array}$ & $\begin{array}{l}\text { Erwachsene; } \\
\text { klinisch/nichtklinisch }\end{array}$ \\
\hline
\end{tabular}


Tab. 1 Instrumente zur Erfassung der Lebensqualität (Fortsetzung)

\begin{tabular}{|c|c|c|c|}
\hline Name & Art der Erfassung & Erfasste Kategorien & Einsatzgebiete \\
\hline Short Form 36 (SF-36) & $\begin{array}{l}\text { Fragebogen oder Interview } \\
\text { zur Selbsteinschätzung; } \\
\text { Version zur Fremdeinschät- } \\
\text { zung; } \\
\text { dichotomes Antwort- } \\
\text { verfahren }\end{array}$ & $\begin{array}{l}36 \text { Items; } \\
8 \text { Dimensionen: körperliche Funktionsfähigkeit, körper- } \\
\text { liche Rollenfunktion, Schmerz, allgemeine Gesundheits- } \\
\text { wahrnehmung, Vitalität, soziale Funktionsfähigkeit, } \\
\text { emotionale Rollenfunktion, psychisches Wohlbefinden }\end{array}$ & Ab 14. Lebensjahr \\
\hline $\begin{array}{l}\text { Social Interview } \\
\text { Schedule (SIS) }\end{array}$ & Interview & $\begin{array}{l}39 \text { Items; } \\
3 \text { Skalen: objektive Lebensbedingungen, } \\
\text { "Social management" }{ }_{\text {,', satisfaction“ }}\end{array}$ & Ab 16. Lebensjahr \\
\hline $\begin{array}{l}\text { Satisfaction With Life } \\
\text { Scale (SWLS) }\end{array}$ & $\begin{array}{l}\text { 1-dimensionales Selbst- } \\
\text { beurteilungsverfahren; } \\
\text { 7-stufiges Antwortverfahren }\end{array}$ & 5 Items & $\begin{array}{l}\text { Erwachsene; } \\
\text { Forschung und Praxis }\end{array}$ \\
\hline $\begin{array}{l}\text { WHO-Instrumente zur } \\
\text { Erfassung der Lebens- } \\
\text { qualität (WHOQOL- } \\
\text { 100/-BREF) }\end{array}$ & $\begin{array}{l}\text { Fragebogen zum Selbst- } \\
\text { ausfüllen }\end{array}$ & $\begin{array}{l}100 \text { Items (BREF: } 26 \text { Items); } \\
6 \text { Skalen (BREF: } 4 \text { Skalen): physische Lebensqualität, } \\
\text { psychische Lebensqualität, Unabhängigkeit, soziale Be- } \\
\text { ziehungen, Umwelt, Religion/Spiritualität }\end{array}$ & $\begin{array}{l}\text { Erwachsene, Gesunde/Kranke; } \\
\text { Forschung, Studien, Outcome- } \\
\text { Indikatoren, Qualitätssicherung }\end{array}$ \\
\hline
\end{tabular}

wachsenen angewendet werden kann. - Tab. 1 soll dabei helfen, das passende Instrument für die eigene Forschung im deutschsprachigen Raum zu finden.

\section{Fazit für die Praxis}

\section{- Die Einbeziehung der Verfahren zur Lebensqualitätsmessung ist in der medizinischen Forschung längst etab- liert. \\ - Da die meisten Instrumente bis da- to nur subjektive Parameter einschlie- ßen, wäre es sehr wünschenswert, Messinstrumente zu entwickeln, die zusätzlich auch objektive Parameter einschließen. \\ - Die Gesundheitsförderung möchte jedem Menschen eine maximale Le- bensqualität ermöglichen. Dieses Ziel rückt durch den Einsatz der aufge- führten Messinstrumente näher. Die Auswahl des passenden Instruments ist aber bei jeder einzelnen Studie aufs Neue eine große Herausforde- rung.}

\section{Korrespondenzadresse}

\section{Kramer}

Abteilung für Gynäkologische Endokrinologie und Reproduktionsmedizin, Frauenklinik, Inselspital Bern

Effingerstr. 102, 3010 Bern

Schweiz

luisa.kramer@students.unibe.ch

\section{Einhaltung ethischer Richtlinien}

Interessenkonflikt. L. Kramer, J. Füri und P. Stute geben an, dass kein Interessenkonflikt besteht.

Dieser Beitrag beinhaltet keine Studien an Menschen oder Tieren.

\section{Literatur}

1. WHO (2005) Bangkok Charta für Gesundheitsförderung in einer globalisierten Welt. S 1-6

2. Barofsky I (2003) Patients' rights, quality of life, and health care system performance. Qual Life Res 12:473-484

3. Rose M (2003) Messung der Lebensqualität bei chronischen Erkrankungen. Habilitationsschrift

4. Campbell A, Converse PE, Rodgers WL (1976) The quality of American life. Russell Sage Foundation, New York, S 411

5. Wolfensberger W (1994) Let's hang up „,Quality of life" as a hopeless term. In: Goode D (Hrsg) Quality of life for persons with disabilities. International perspectives and issues. Brookline Books, Cambridge/MA, S 285-321

6. Schumacher J, Klaiberg A, Brähler E (2003) Diagnostische Verfahren zu Lebensqualität und Wohlbefinden. Hogrefe, Göttingen, S 329

7. Mattejat F, Jungmann J, Meusers M et al (1998) Das Inventar zur Erfassung der Lebensqualität bei Kindern und Jugendlichen. S 1-16

8. Kuyken W (1995) The World Health Organization Quality of Life Assessment (WHOQOL): position paper from the Worlds Health Organization. Trial 41

9. Prutkin JM (2002) A history of quality of life measurements. Doktorarbeit

10. Radoschewski M (2000) Gesundheitsbezogene Lebensqualität - Konzepte und Maße. Bundesgesundheitsbl 43:165-189

11. Ravens-Sieberer U, Cieza A (2000) Lebensqualität und Gesundheitsökonomie in der Medizin. Ecomed, S 466

12. Daig I, Lehmann A (2007) Verfahren zur Messung der Lebensqualität. Psychologie. Schweiz Z Psychol Anwend 16:5-23
13. Pöthig D, Stute $P$ (2010) Vitalitätsdiagnostik: Gesundheit erhalten statt Krankheit verwalten. Geburtshilfe Frauenheilkd 509-511

14. Preamble to the Constitution of the World Health Organization as adopted by the International Health Conference, New York, 19-22 June, 1946; signed on 22 July 1946 by the representatives of 61 States (Official Records of the World Health Organization, no. 2, p. 100) and entered into force on 7 April 1948

15. Bullinger M, Siegrist J, Ravens-Sieberer U (2001) Lebensqualitätsforschung aus medizinpsychologischer und -soziologischer Perspektive. Hogrefe, Göttingen, S 388

16. Bech $P$ (1995) Quality of life measurement in the medical setting. Eur Psychiatry 10 (Suppl 3):83s$85 \mathrm{~s}$

17. Baker R (1999) Quality of life assessment in clinical trials: methods and practice. J R Soc Med 92:49

18. Gill TM (1995) Quality of life assessment: values and pitfalls. J R Soc Med 88:680-682

19. Herschbach $P$ (2000) Der Nutzen der Lebensqualitätsdiskussion für die Patienten. In: Ravens-Sieberer U, Cieza A (Hrsg) Lebensqualität und Gesundheitsökonomie in der Medizin. S 466

20. Muldoon MF, Barger SD, Flory JD, Manuck SB (1998) What are quality of life measurements measuring? Br Med J 316:542-545

21. Güthlin C (2006) Die Messung gesundheitsbezogener Lebensqualität: ausgewählte psychometrische Analysen und Anwendungsprobleme. Dissertation

22. Crosby R (2003) Defining clinically meaningful change in health-related quality of life. J Clin Epidemiol 56:395-407 